\title{
Electrospun Janus nanofibers loaded with a drug and inorganic nanoparticles as an effective antibacterial wound dressing
}

Jinke Yang, 1 Ke Wang, ${ }^{2}$ Deng-Guang Yu, 1, "Yaoyao Yang, 1

Sim Wan Annie Bligh,2, * Gareth R Williams 3

School of Materials Science \& Engineering, University of Shanghai for Science and Technology, Shanghai 200093, China

School of Health Sciences, Caritas Institute of Higher Education, 2 Chui Ling

Lane, Tseung Kwan O, New Territories, Hong Kong 999077, China

UCL School of Pharmacy, University College London, 2939 Brunswick Square, London WCIN IAX, UK

Declarations of interest: none.

* Corresponding authors:

Prof. Deng-Guang Yu 
School of Materials Science \& Engineering

University of Shanghai for Science and Technology

516 Jungong Road, Shanghai 200093, P.R. China

Tel/Fax: +86-21-55270632

E-mail: ydg017@usst.edu.cn

Prof. S. W. Annie Bligh

E-mail: abligh@cihe.edu.hk

\section{Graphical abstract}
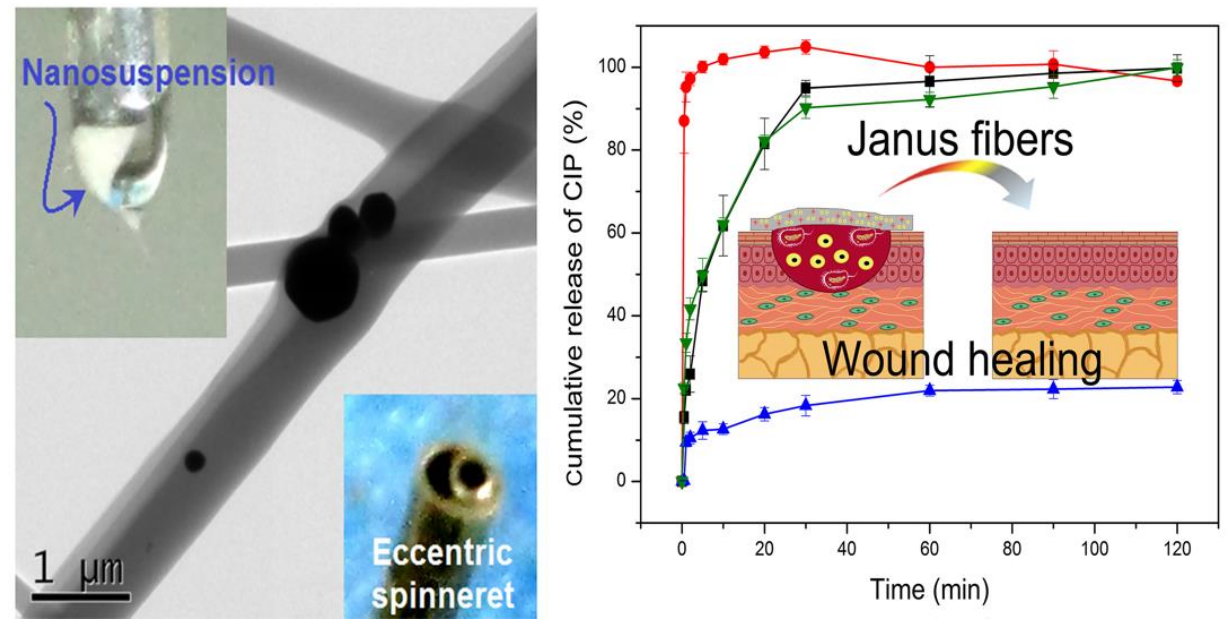


\section{Highlights}

- A side-by-side electrospinning process was conducted using a nanosuspension as a working fluid fluid

- Hydrophilic polyvinylpyrrolidone and hydrophobic ethyl cellulose are combined into Janus fibers

- Ciprofloxacin and $\mathrm{Ag}$ nanoparticles are encapsulated into the Janus nanofibers

- The Janus nanofibers showed an improved performance against bacteria 


\section{Abstract}

The most important property of a wound dressing is its anti-bacteria performance. Although electrospun nanofibers are frequently demonstrated to be potent candidates as wound dressings, no Janus fibers have been explored for this popular application. In this study, a Janus wound dressing composed of polyvinylpyrrolidone (PVP) and ethyl cellulose (EC) polymer matrices was prepared via a side-by-side electrospinning process, in which ciprofloxacin (CIP) and silver nanoparticles (AgNPs) were loaded in the two sides. A homemade acentric spinneret was exploited to maintain a continuous preparation process. Scanning and transmission electron microscope results demonstrated that the Janus fibers had a uniform and cylindrical morphology with a clear Janus structure, and AgNPs distributed in one side. X-ray diffraction patterns suggested that drug was present in the fibers in an amorphous state owing to rapid drying and its good compatibility with PVP, which was verified by infrared spectroscopy. In vitro tests showed that over 90\% of CIP was released within the first 30 
minutes, ensuring a strong antibacterial effect at the initial stages of wound healing. The Janus fibers were demonstrated to have good bactericidal activity against the growth of both Gram-positive S. aureus and Gram-negative E. coli. The PVP-CIP/EC-AgNPs Janus fibers could thus be a promising candidate for effective wound dressings. This work paves a new way for creating Janus structure-based advanced functional nanomaterials.

\section{Keywords}

Side-by-side electrospinning; Janus nanofiber; Organic/inorganic hybrids; Wound dressing; Anti-bacteria; Synergistic effect

\section{Introduction}

In human body, the skin is the biggest organ. It is an effective barrier to pathogens. It plays important roles in protection, immunization, regulation of body temperature and sensory function [1]. However, as the interface between other organs of the body and the environment, skin is easily injured. With skin injuries, one of the key issues is bacterial infection [2], which makes the wound healing process very complex [3-7]. Thus, wound dressings with high 
antibacterial effect are always popular both in the academic studies and commercial applications [8].

In this nano era, wound dressings composed of nanomaterials are drawing increasing attention to meet the large demands from the commercial markets and overcome the drawbacks of traditional wound dressings [9]. Fundamentally, what an ideal dressing must provide are the moist wound environment, the ability to remove wound exudates, and tissue regeneration promotion. But first of all, it should have biocompatibility with good antimicrobial performances [10].

Almost all kinds of new advanced material processing technologies have once been introduced in the potential applications as wound dressings, and there are many review articles on this topic in literature [1 1]. Electrospinning, as a peer method of electrospraying for generating nanofibers, is extending its applications in every branch in biomedical engineering [12-15]. Among different types of pharmaceutical applications, wound dressing from electrospun medicated nanofibers holds the great promises to become commercial products [16]. The unique properties of electrospun nanofibers such as small diameter, huge surface, large porosity and the random 3-D web deposition micro-structures make them good candidates as wound dressings naturally. Thus, a wide variety of polymeric matrices have been investigated for this application based on the single-fluid blending electrospinning and the 
resulted monolithic nanofibers. However, although the electrospun core-shell nanostructures have been applied in some important areas, such as energy [17], electrospun wound dressings from the double-fluid coaxial electrospinning are still very limited [18]. What is more, none reports could be found on the applications of electrospun Janus fibers as wound dressing up to date.

This situation should have a close relationship with the developments of electrospinning itself. To implement a monoaxial electrospinning, the prerequisite is that the working fluid must be electrospinnable [15]. The working fluid is often composed of a host filament-forming polymeric matrix and a guest active ingredient, which endows the nanofibers the desired functional performance. Through this way, many pharmaceutical active ingredients have been incorporated into the electrospun polymeric nanofibers to improve their antibacterial effects [18]. As for the coaxial electrospinning, there are two working fluids that are treated simultaneously for forming the core-shell nanostructures [19]. In a typical traditional coaxial process, the shell fluid must be electrospinnable to ensure a successful working process for creating the core-shell solid structures [20]. Recently, a modified coaxial electrospinning is reported, in which unspinnable fluids can be exploited as the shell fluids, and solid core-shell structure, nanocoating and monolithic nanofibers can all be prepared using this technique [21]. The coaxial and modified coaxial process are more difficult than the traditional blend 
electrospinning in terms of implementation and manipulation because a series of new issues are involved, such as the core-to-shell fluid flow rate ratio, their compatibility, and their matching behaviors in the electrical field. However, the side-by-side electrospinning is even more difficult than the two types of coaxial processes and even the tri-fluid coaxial electrospinning [22]. This is because that all the different kinds of coaxial processes have only one fluid directly contacting the environmental electrical field, regardless of two, three or even four working fluids treated at the same time. In contrast, the two working fluids of side-by-side electrospinning are simultaneously under the electrical fields with the same negative or positive charges, which makes it very easy for them to departure from each other, and thus in turn results in the failure of creating integrated Janus structures [23]. This should be the most important reason that the reports on Janus nanofibers are still very limited, let alone Janus nanostructures for wound dressing applications.

Most recently, an elaborate side-by-side electrospinning was reported for effective creation of integrated Janus nanostructure, in which an acentric spinneret was developed to eliminate the repulsion between the two side-byside working fluids [24]. Based on this advanced method, here we hypothesize that new wound dressing from Janus nanofibers can be developed. The new Janus wound dressing should, on the one hand, contribute to hemostasis, increase the absorptivity of wound exudate and the flexibility of dressing design, keep the wound environment moist and allow the permeation of 
oxygen and water. This is because the Janus fibers have the large specific surface area volume ratio, high porosity, and similar matrix morphology with human extracellular matrix, just as the monolithic nanofiber mats from the blending electrospinning. On the other hand, different kinds of active ingredients can be simultaneously loaded into the Janus structure and the advantage of Janus structure with both sides contacting their environment can be explored for a synergetic effect on the desired functional performances, such as anti-bacteria. Thus, electrospun Janus nanofiber can bring together multiple disciplines and accelerate the development of novel functional nanomaterials (Schematic 1).

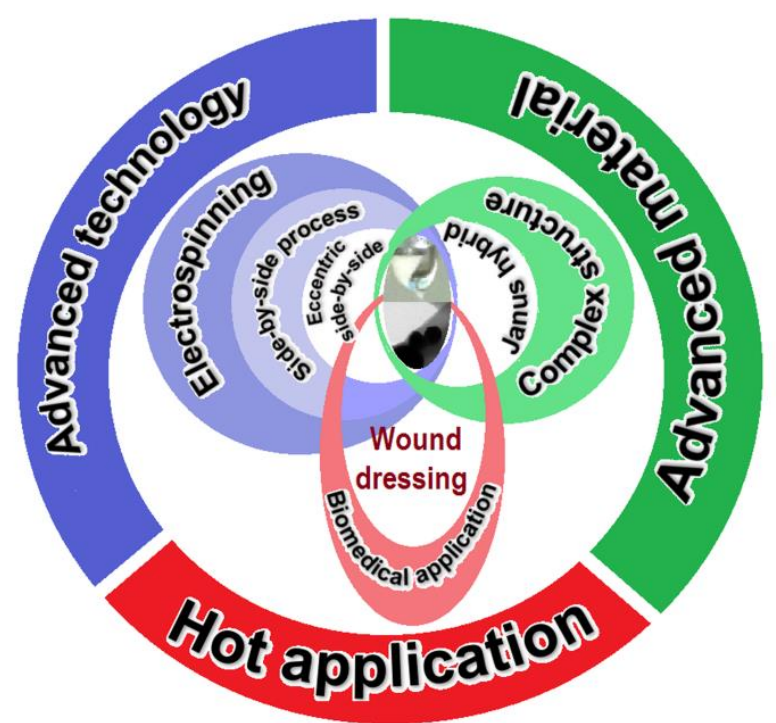

Schematic 1. The convergent point based on electrospun Janus structures.

During the past two decades, both natural and synthetic polymers have been tried their potential applications as electrospun wound dressing matrices. The typical natural polymers include gelatin [25], fibrin [26], zein 
[27], cellulose and its derivatives [28, 29]. The synthetic polymers have a larger number than the natural ones, such as polyvinyl pyrrolidone (PVP) [30-32], polyvinyl alcohol (PVA) [33,34], polylactic acid (PLA) [35,36], polycaprolactone (PCL) [37-39], polyurethane (PU) [40], and polyethylene oxide (PEO) [41]. Among these polymers, PVP, as a hydrophilic pharmaceutical excipient, is one of the most extensively exploited polymeric matrices [42]. Ethyl cellulose (EC), a biocompatible and non-biodegradable polymer, is also one of the most popular pharmaceutical excipient, whose nanofibers had extremely good permeability [43].

There are many kinds of drug candidates that can be applied to wounds healing. Among them, ciprofloxacin (CIP) is a very popular antibiotic. Its spectrum of antibacterial includes gram-positive and gram-negative bacteria, so it is effective on a wide range of infections [44]. Several studies have demonstrated that CIP plays a great role in the process of wound healing [4547]. Silver nanoparticles (AgNPs) are also known to have a broad range of antimicrobial activity since ancient time. And the ability of them to prevent bacterial infections of the wound areas through destroying the membrane and DNA of bacteria has been demonstrated [48-50]. There are growing number of researches about the applications of AgNPs in wound dressings, and all the results in promoting wound healing are positive [2, 51-53]. Based on the above-mentioned knowledge, here we demonstrate a proof-of-concept investigation using side-by-side electrospinning. A new type of PVP-CIP//EC- 
AgNPs Janus nanofibers are created as wound dressings. The morphology, structure, compatibility and antibacterial effects of the medicated Janus fibers were investigated in detail, and the mechanism for the synergetic effects of two active ingredients in the Janus structure is proposed.

\section{Materials and methods}

\subsection{Materials}

PVP K90 $\left(\bar{M}_{\mathrm{W}}=1,300,000\right)$ and CIP were purchased from Shanghai Aladdin Chemistry Co. Ltd. (Shanghai, China). EC (8.0 cPa s to $10.0 \mathrm{cPa}$ s), ethanol, acetone and acetic acid were obtained from Sinopham Chemical Reagent Co. Ltd. (Shanghai, China). AgNPs were obtained from Wuhan Lullaby Pharmaceutical Chemical Co. Ltd. (Wuhan, China). All the other chemicals used in this work were analytical grade, and water was doubly distilled before use.

\subsection{Electrospinning}

The solvent mixture A and B were composed of ethanol and acetic acid (9:1 in volume), and ethanol and acetone (1:1 in volume), respectively. Based on solvent mixture $\mathrm{A}$, two electrospinnable solutions were prepared as follows: one blank solution was composed of $0.8 \mathrm{~g}$ PVP in $10 \mathrm{~mL}$ solvent, the other contained $0.08 \mathrm{~g} \mathrm{CIP}$ and $0.8 \mathrm{~g}$ PVP in $10 \mathrm{~mL}$ solvent. Similarly, based on solvent mixture B, two working fluids were prepared as follows: one blank solution was composed of $1.4 \mathrm{~g} \mathrm{EC}$ in $10 \mathrm{~mL}$ solvent, the other nanosuspension contained $0.5 \mathrm{~g}$ AgNPs and $1.4 \mathrm{~g} \mathrm{EC}$ in $10 \mathrm{~mL}$ solvent. 
In this work, two monolithic and four Janus fibers were fabricated. Details about the parameters for implementing the single and side-by-side electrospinning processes are concluded in Table 1.

Table 1. Parameters of the electrospinning processes.

\begin{tabular}{lccccccc}
\hline & & \multicolumn{3}{c}{ PVP side } & \multicolumn{5}{c}{ EC side } \\
\cline { 2 - 7 } No. & \begin{tabular}{c} 
Process \\
\cline { 3 - 7 }
\end{tabular} & $\begin{array}{c}\text { Flow rate } \\
\mathrm{b}\end{array}$ & PVP c & CIP c & $\begin{array}{c}\text { Flow rate } \\
\mathrm{b}\end{array}$ & EC c & AgNPs c \\
\hline F1 & Single & 1.0 & 0.8 & 0.08 & -- & -- & -- \\
F2 & Single & - & - & - & 1.0 & 1.4 & 0.5 \\
F3 & SS & 1.0 & 0.8 & 0 & 1.0 & 1.4 & 0 \\
F4 & SS & 1.0 & 0.8 & 0.08 & 1.0 & 1.4 & 0 \\
F5 & SS & 1.0 & 0.8 & 0 & 1.0 & 1.4 & 0.5 \\
F6 & SS & 1.0 & 0.8 & 0.08 & 1.0 & 1.4 & 0.5 \\
\hline
\end{tabular}

a Single and SS denote single-fluid and side-by-side electrospinning, respectively.

b Flow rate in $\mathrm{mL} / \mathrm{h}$.

c Concentration in $\mathrm{g} / \mathrm{mL}$.

Two syringe pumps (KDS100 and KDS200, Cole-Parmer, IL, USA) and a high-voltage power supply (ZGF $60 \mathrm{kV} / 2 \mathrm{~mA}$, Wuhan Huatian Electric Power Automation Co., Ltd, Wuhan, China) were used for driving the two working fluids. The fluids were placed into two $10 \mathrm{~mL}$ plastic syringes, which were fixed on two pumps and were guided to a homemade acentric spinneret. The fluid flow rates of both sides were $1.0 \mathrm{~mL} / \mathrm{h}$. The applied voltage between the 
needle and the grounded collector (a flat piece of aluminum foil) was fixed at 10.0 kV after optimization. The collection distance was $15 \mathrm{~cm}$. The ambient temperature and relative humidity were $22 \pm 3 \circ \mathrm{C}$ and was $50 \pm 5 \%$, respectively. The fibers were stored in an oven of $60{ }^{\circ} \mathrm{C}$ for $24 \mathrm{~h}$ to remove residual solvent after electrospinning.

\subsection{Characterization}

\subsubsection{Morphology and structure}

The fibers were gold sputter-coated for $100 \mathrm{~s}$ under nitrogen atmosphere. Their morphology was observed using a field emission scanning electron microscopy (SEM, FEI Quanta450FEG, USA). The average diameters of the fibers were estimated using ImageJ software (National Institutes of Health, USA) by analyzing over 100 fibers in SEM images. The Janus structures of fibers were

verified using a transmission electron microscope (TEM, JEM 2100F, JEOL, Tokyo, Japan).

\subsubsection{Physical status and compatibility}

The X-ray diffraction (XRD) analysis was conducted by a Bruker X-ray diffractometer Bruker-AXS with $\mathrm{Cu} \mathrm{K} \alpha$ radiation (Karlsruhu, Germany), and the patterns were collected over the $2 \theta$ range $10-60^{\circ}$. The voltage applied was $40 \mathrm{kV}$ and the current was $30 \mathrm{~mA}$. 
Attenuated total reflectance-Fourier transform infrared (ATR-FTIR) spectroscopy was undertaken using a Spectrum 100 FTIR Spectrometer (PerkinElmer, Billerica, USA) at a range of $500-4000 \mathrm{~cm}^{-1}$ at a resolution of 2 $\mathrm{cm}^{-1}$.

\subsection{Functional applications}

\subsubsection{In vitro dissolution tests}

In vitro dissolution tests were conducted according to the Chinese Pharmacopoeia (2015 Ed.). $100 \mathrm{mg}$ CIP powders or fibers containing $100 \mathrm{mg}$ drug were immersed in $450 \mathrm{~mL}$ phosphate buffer saline (PBS, pH7.0, $0.1 \mathrm{M})$ at $37{ }^{\circ} \mathrm{C}$. At predetermined time points, $5.0 \mathrm{~mL}$ aliquot was withdrawn and $5.0 \mathrm{~mL}$ of fresh PBS was added. The amounts of CIP released were analyzed at $\lambda_{\max }=276 \mathrm{~nm}$ using a UV-vis spectrophotometer (UV-2102PC, Unico Instrument Co. Ltd., Shanghai, China). Experiments were repeated 3 times and the results are reported as mean \pm S.D.

\subsubsection{Antibacterial activity}

Antibacterial activities of the nanofibers were examined with the disc diffusion method according to the US Clinical and Laboratory Standards Institute. In the experiments, S. aureus (Gram-positive, ATCC 27853) and E. coli (Gram-negative, ATCC 25922) were selected as representative microorganisms, and cultured on nutrient broth agar plates. The size of the circular fiber samples was $5 \mathrm{~mm}$ in diameter and sterilized by pressure steam. The surfaces of agar plates were uniformly daubed by $5 \times 105$ colony forming 
units (CFUs)/mL of the bacterial suspension 3 times. All the dishes were cultured at $37{ }^{\circ} \mathrm{C}$ for $72 \mathrm{~h}$. Inhibition zones were measured after $24 \mathrm{~h}$ and 72 h.

\subsection{Statistical analysis}

The experimental data are presented as mean \pm SD. The results of antibacterial activity tests were analyzed using one-way ANOVA. The difference was considered significant when $p$ (probability) value was less than 0.05 .

\section{Results and discussion}

\subsection{Side-by-side electrospinning}

A side-by-side electrospinning system is similar with a typical single-fluid or a coaxial one. Shown in Fig. 1, it includes a high-voltage power supply to provide electrostatic energy, two syringe pumps to drive the working fluids, a side-by-side spinneret and a grounded fiber collector. Side-by-side electrospinning, a peer of coaxial electrospinning, treats two working fluids simultaneously for creating Janus fibers, in which two different sides are bound together and both directly contact the environment. As one of the most difficult challenges in electrospinning filed [24], very limited articles have been published about this advanced nanotechnology. Traditionally, two parallel metal capillaries comprise the side-by-side spinneret, which is very difficult to manipulate due to the frequent separation of two same charge working 
fluids. Some kinds of modified techniques have been reported to try to resolve this issue. For example, a Chinese patent has demonstrated the usefulness of a round side-by-side spinning head with an internal bisection capillary in creating Janus fibers [54]. Another acentric spinneret for implementing sideby-side electrospinning was reported both in a patent [55] and two publications [24,56], which has an elliptical outer nozzle containing an inner round side and an inner crescent side. In this study, an acentric spinneret was developed to conduct the side-by-side electrospinning processes for preparing the Janus nanostructures.

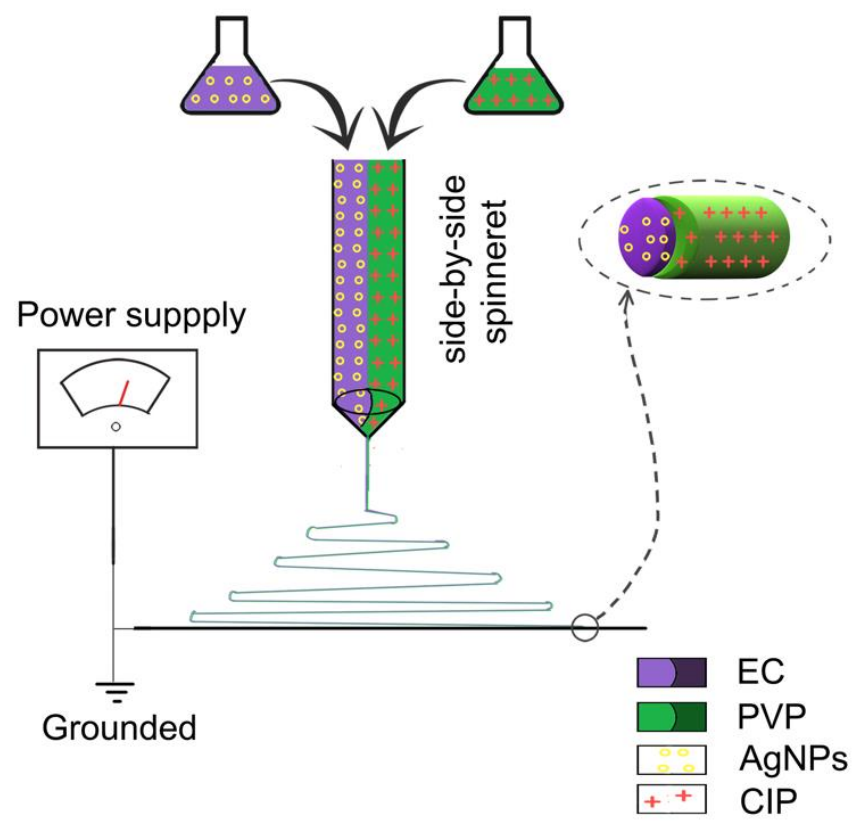

Fig. 1. A schematic diagram of the side-by-side electrospinning process.

Shown in Fig. 2 are three digital pictures of the developed acentric spinneret taken from different angles. Compared to a traditional side-by-side spinneret consisting of two parallel metal capillaries, it shows several 
advantages in ensuring the creation of integrated Janus fibers. Fig. $2 \mathrm{a}$ is a full view of the spinneret and Fig. $2 \mathrm{~b}$ and $2 \mathrm{c}$ give the details about the elaborate designs about the elliptical nozzle. In Fig. 2 b, the round side capillary slightly projected out the crescent side about $0.2 \mathrm{~mm}$, which helps to retard the possible diffusion between the two working fluids. In Fig. 2c, the two sides compose a whole round outer shape, ensuring an effective electro-hydrodynamic interaction. Meanwhile, the two side fluids have a certain length of arc to bind with each other when they were introduced into the electrical field, which is important to keep the two fluids from departure during the electrical drawing.
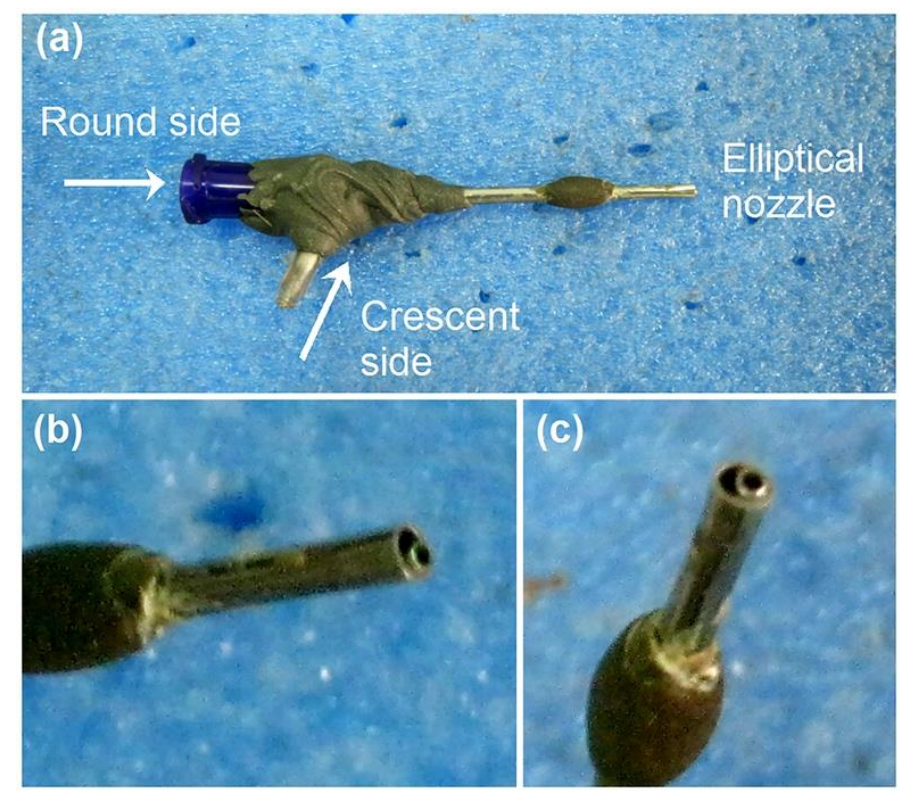

Fig. 2. A digital picture of the acentric side-by-side spinneret: (a), (b) and (c) were taken from different angles.

The homemade electrospinning system, the working processes of singlefluid and side-by-side electrospinning are shown in Fig. 3. The 
electrospinning system exhibited in Fig. $3 \mathrm{a}$ is composed of the typical four components: two pumps, a collector, a spinneret, and a power supply. Fig. 3b tells how to connect the spinneret with the working fluids and the high voltage supply. A syringe containing the EC-AgNPs suspensions fixed on a pump was direct inserted into the acentric spinneret, by which the nanosuspensions were guided to the round side of the spinneret nozzle. The other side PVP-CIP solutions loaded into another syringe and fixed on another pump were driven to the crescent side exit of the spinneret nozzle. High voltage was transferred to the working fluids through an alligator clip.

In the present job, both side working fluids had fine electrospinnability. When the pump driving the round side EC-AgNPs suspension was turned off, a typical electrospinning process for creating nanofibers $\mathrm{F} 1$ was recorded in Fig. 3c. A transparent Taylor cone (the inset) emitted a straight fluid jet, which was followed by bending and whipping loops. Similarly, when the pump driving the crescent side PVP-CIP solution was switched off, a typical electrospinning process for creating nanofibers F2 was recorded in Fig. 3d. Its upper-right inset shows a representative opaque Taylor cone. However, the suspensions were easy to cling on the spinneret, to enlarge the Taylor cone (the upper-left inset), and finally to clog the exit of nozzle, which is also reported in literature [22]. Thus, manual removement of the excessive semi-solid substance around the nozzle was needed for a continuous preparation of monolithic AgNPsloaded EC nanofibers F2. 
When both pumps were turned on and regulated to a flow rate of $1.0 \mathrm{~mL} / \mathrm{h}$, the side-by-side working processes were robust and continuous (Fig. 3e). The upper-left inset shows a compound Janus Taylor cone with the one clear side transparent solution and the other side opaque nanosuspension. This phenomenon suggested that the both sides had fine compatibility, and what is more, the electrospinnable PVP-CIP solutions could help to eliminate the clogging possibility from the EC-AgNPs side, and thus to ensure a whole and continuous preparation.

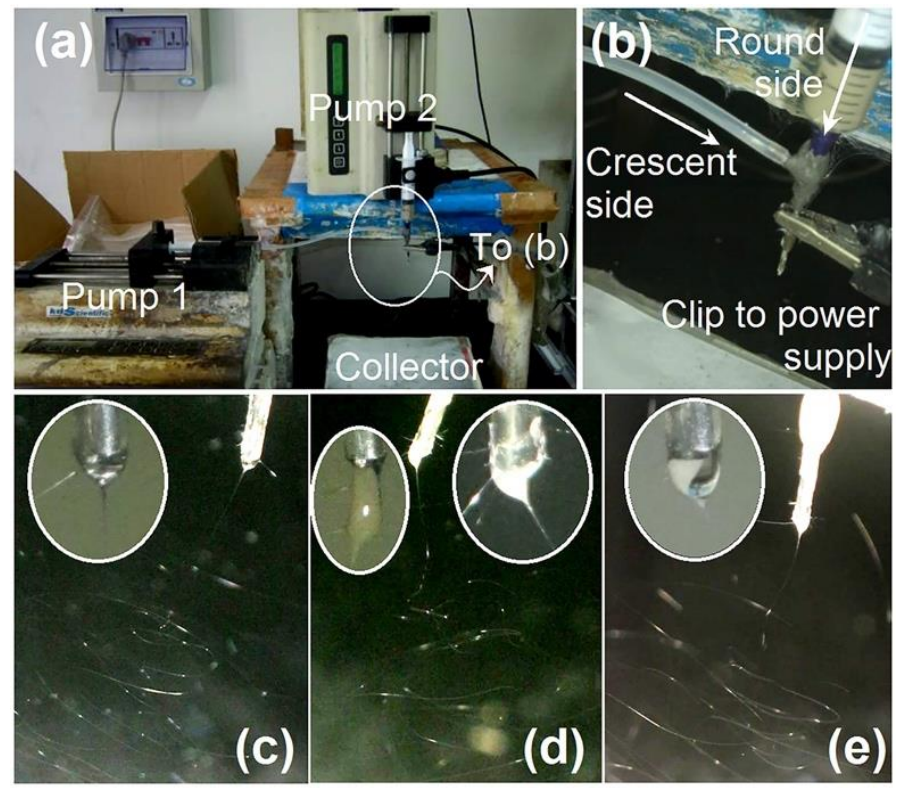

Fig. 3. The electrospinning process: (a) the homemade electrospinning system; (b) the connection between the spinneret with the working fluids and power supply; (c) The preparation of nanofibers F1 consisting of PVP-CIP from the crescent side, with an inset of a transparent Taylor cone; (d) The preparation of nanofibers F2 consisting of EC-AgNPs from the round side, with an inset of the opaque nanosuspension Taylor 
cone; (e) A side-by-side process for preparing Janus fibers F5 with an inset of opaque compound Taylor cone.

\subsection{Fiber morphology}

The SEM images about the morphologies of nanofibers F1 to F6 are shown in Fig. 4. The monolithic PVP-CIP nanofibers F1 (Fig. 4a) had a fine linear morphology without the beads-on-a-string or spindles-on-a-string phenomena with an average diameter of $0.78 \pm 0.18 \mu \mathrm{m}$. Meanwhile, the nanofiber surfaces are smooth without any discerned particles on them, giving a hint that no solid phase separation happened during the preparation processes due to the good compatibility between PVP and CIP. In contrast, the electrospun EC-AgNPs fibers F2 had a typical spindles-on-line morphology (Fig. 4b), suggesting that the suspensions had a poor electrospinnability. On the surfaces of those spindles, some bright dots can be observed, which should be the presence of AgNPs.

Four kinds of Janus nanofibers F3, F4, F5 and F6 were conceived and prepared, with loaded functional ingredients of none, CIP in the PVP side, AgNPs in the EC side, and CIP in PVP side and AgNPs in EC side, respectively. All the four kinds of Janus nanofibers have good linear morphology with fewer beads-on-a-string on them (Fig. 4c, 4d, $4 \mathrm{e}$ and $4 \mathrm{f}$, respectively), indicating that the two side working fluids had good compatibility. During the side-by- 
side electrospinning processes, the PVP-CIP side solutions should have help the EC-AgNPs suspensions to be drawn under the electrical field in a matched manner, thus the spindles in fibers F2 disappeared here. Compared with the smooth surfaces of Janus fibers F3 and F4, Janus fibers F5 and F6 had some bright dots on them due to the presence of AgNPs. Meanwhile, their average diameters of $0.82 \pm 0.16 \mu \mathrm{m}$ for $\mathrm{F} 5$ and $0.84 \pm 0.24 \mu \mathrm{m}$ for $\mathrm{F} 6$ are slightly larger than those of Janus fibers F3 $(0.73 \pm 0.18 \mu \mathrm{m})$ and F4 $(0.74 \pm 0.19 \mu \mathrm{m})$. 

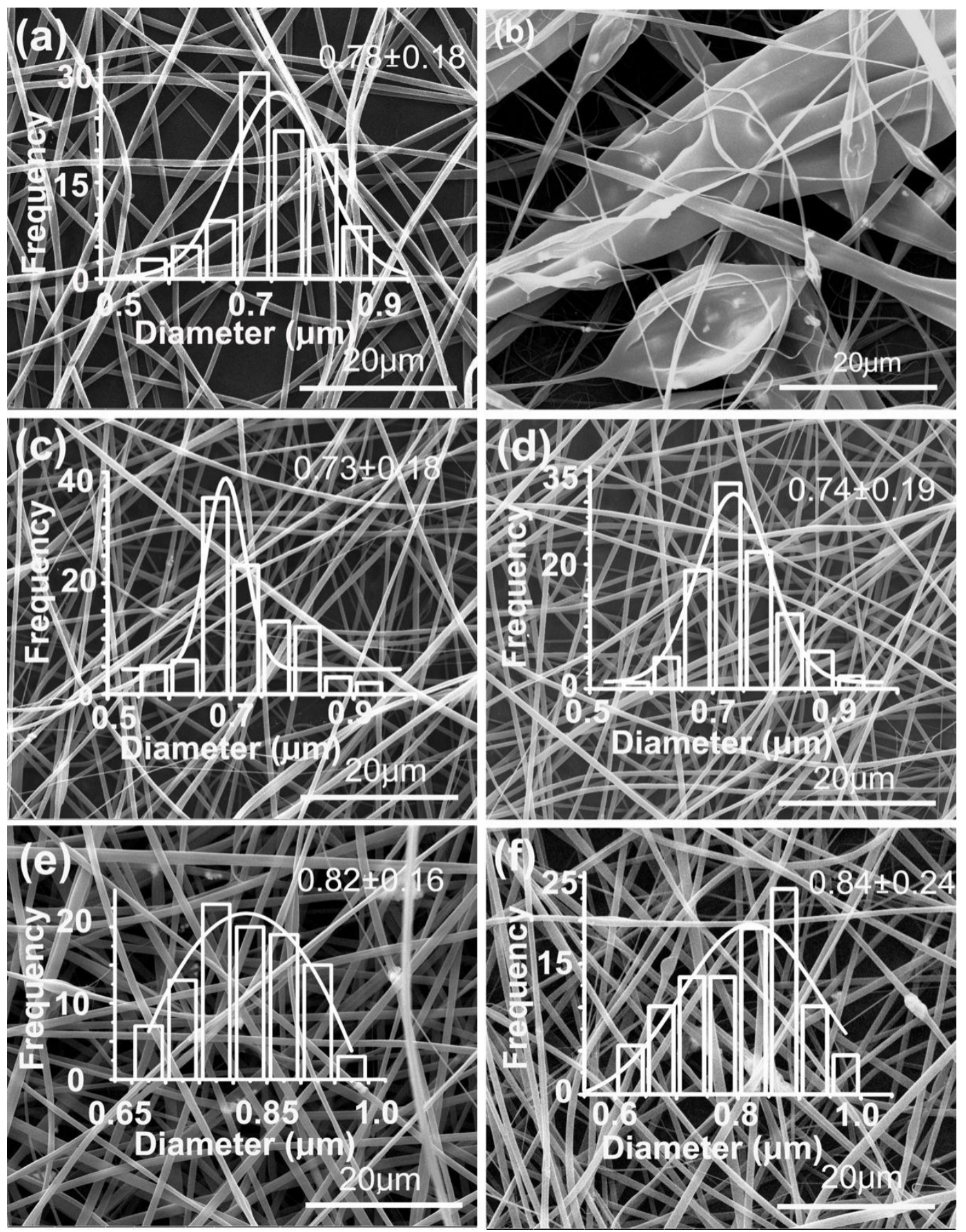

Fig. 4. SEM images and diameter distributions of the nanofibers: (a) F1;

(b) F2; (c) F3; (d) F4; (e) F5; (f) F6.

A typical TEM image of the F6 formulation is displayed in Fig. 5. A Janus structures with two different sides in the electrospun nanofibers can be observed clearly. The linear section with deeper gray level in the fiber was the 
EC-AgNPs side, in which the black dots of AgNPs were recorded. The section with smaller gray level was the PVP-CIP section, in which no CIP nanoparticles could be discerned. This indicates that the drug CIP were completely converted into an amorphous state, and a monolithic nanocomposite with the guest CIP loaded (homogeneously and most probably in a molecular manner) in the host PVP was successfully fabricated as one side of the Janus nanostructures.

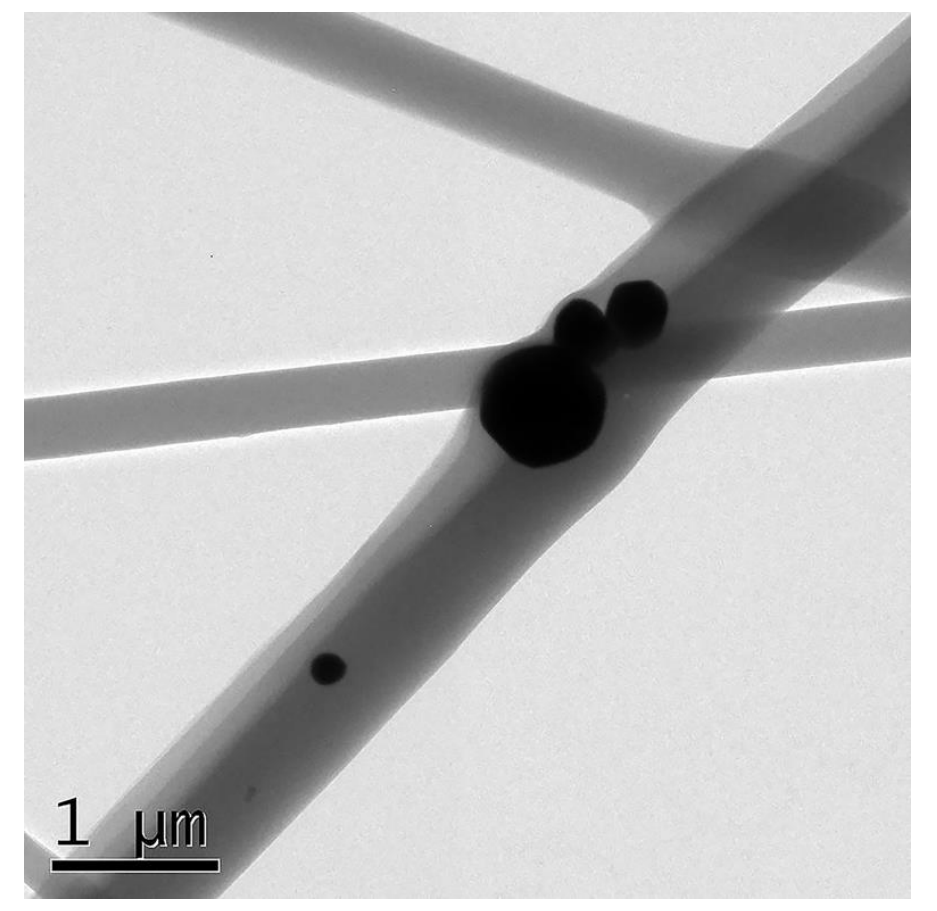

Fig. 5. TEM image of the Janus fibers co-loaded with CIP and AgNPs.

\subsection{Physical form and compatibility of components}

XRD patterns are presented in Fig. 6 . The sharp peaks at around $38^{\circ}$ and $45^{\circ}$ of AgNPs show their two crystal models. The well-defined Bragg reflections of raw CIP powders demonstrate their crystalline nature. The two weak diffraction halos of PVP and EC suggest that these polymers are in amorphous status. In the patterns of F1, F4, and F6 fibers, the characteristic 
peaks of raw CIP powders completely disappeared. Their broad humps of amorphous status in these fibers' patterns indicated that the loaded CIP was fully transformed into an amorphous composite with the filament-forming polymeric matrix. As for the fibers F2, the presence of two characteristic peaks of AgNPs indicates they are organic/inorganic hybrids. In comparison, the peak at $45^{\circ}$ could not be discerned in the XRD patterns of fibers $F 6$ due to their relatively lower content of AgNPs. However, the characteristic peak around $38^{\circ}$ could still be observed (as indicated by circle "A" in Fig. 6), which suggests the co-presence of AgNPs with other components in the Janus nanostructures.

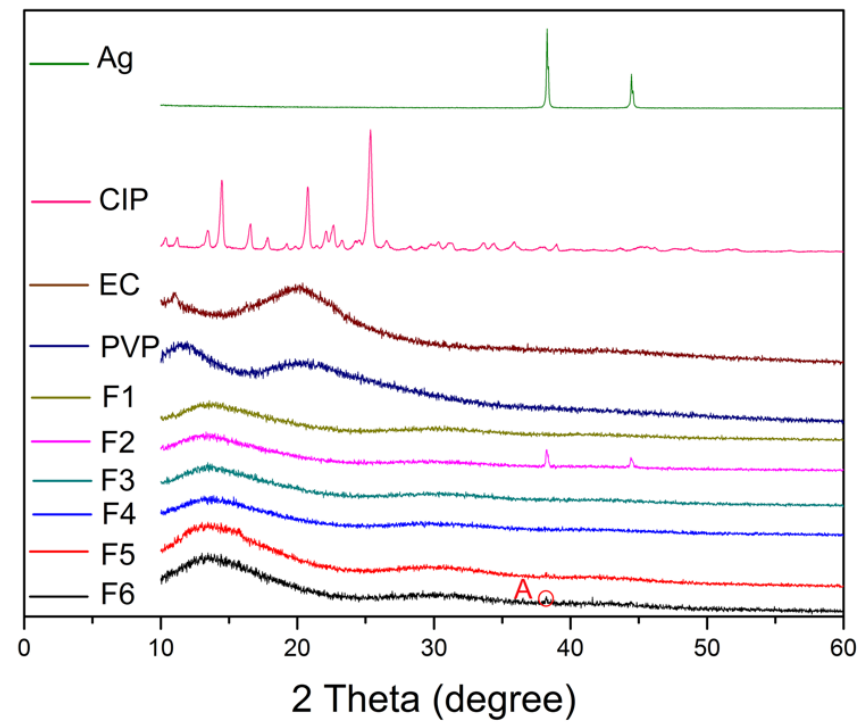

Fig. 6. XRD patterns of the raw materials and their nanofibers.

The ATR-FTIR spectra of the components and the fibers are shown in Fig. 7. Compatibility between the loaded drug and the polymers is crucial for the stability of nanofibers. In the spectrum of PVP, the typical peak at $1659 \mathrm{~cm}^{-1}$ 
is the stretching vibrations of $\mathrm{C}=\mathrm{O}, 1424 \mathrm{~cm}^{-1}\left(=\mathrm{CH}_{2}\right.$ bending $)$ and $1283 \mathrm{~cm}^{-}$ 1 (-C-N- stretching) were also observed. And the very broad band at $3462 \mathrm{~cm}^{-}$ 1 is attributed to the presence of water. As for EC, $2974 \mathrm{~cm}^{-1}$ for C-H stretching, $1375 \mathrm{~cm}^{-1}$ for $\mathrm{C}-\mathrm{H}$ bending and $1054 \mathrm{~cm}^{-1}$ for $\mathrm{C}-\mathrm{O}$ stretching are present in its spectra. The peak at $1614 \mathrm{~cm}^{-1}$ of the CIP is from $\mathrm{C}=\mathrm{O}$ stretching. $1589 \mathrm{~cm}^{-1}$ and $1498 \mathrm{~cm}^{-1}$ are due to the stretching vibrations of the benzene ring, and the peak at $3045 \mathrm{~cm}^{-1}$ corresponds to $\mathrm{O}-\mathrm{H}$ stretching.

In contrast, the nanofibers $\mathrm{F} 1$ have the typical peaks of $1662 \mathrm{~cm}^{-1}$ and $1494 \mathrm{~cm}^{-1}$, which are slightly red shift from $1659 \mathrm{~cm}^{-1}$ of PVP and $1498 \mathrm{~cm}^{-1}$ of CIP, respectively. Besides, almost all the other sharp peaks of CIP have shifted a little, decreased in intensity or even disappeared in the spectra of fibers F1. These phenomena should be attributed the favorite secondary interactions between PVP and CIP. These interactions include hydrogen bonds between the carbonyl groups in PVP and hydroxyl groups in CIP, hydrophobic interactions between the PVP long carbon chain and the CIP benzene rings, and the attractive electrostatic interactions between the positive and negative groups of the PVP and CIP molecules. The situations in the PVP-CIP sides of Janus fibers F4 and F6 should be similar with fibers F1.

Compared with the spectra of EC, fibers F2 to F6 all have the typical C-O stretching signals around $1059 \mathrm{~cm}^{-1}$, suggesting that the presence of EC as polymeric matrix in nanofibers $\mathrm{F} 2$ and as one side in the Janus structures of 
fibers F3, F4 F5 and F6. AgNPs show no responses in the FTIR spectra. The results from XRD and FTIR co-suggested that the Janus fibers F6 were a multiple-component hybrid consisting of a side of PVP-CIP medicated nanocomposite and a side of AgNPs-EC organic-inorganic functional hybrids.
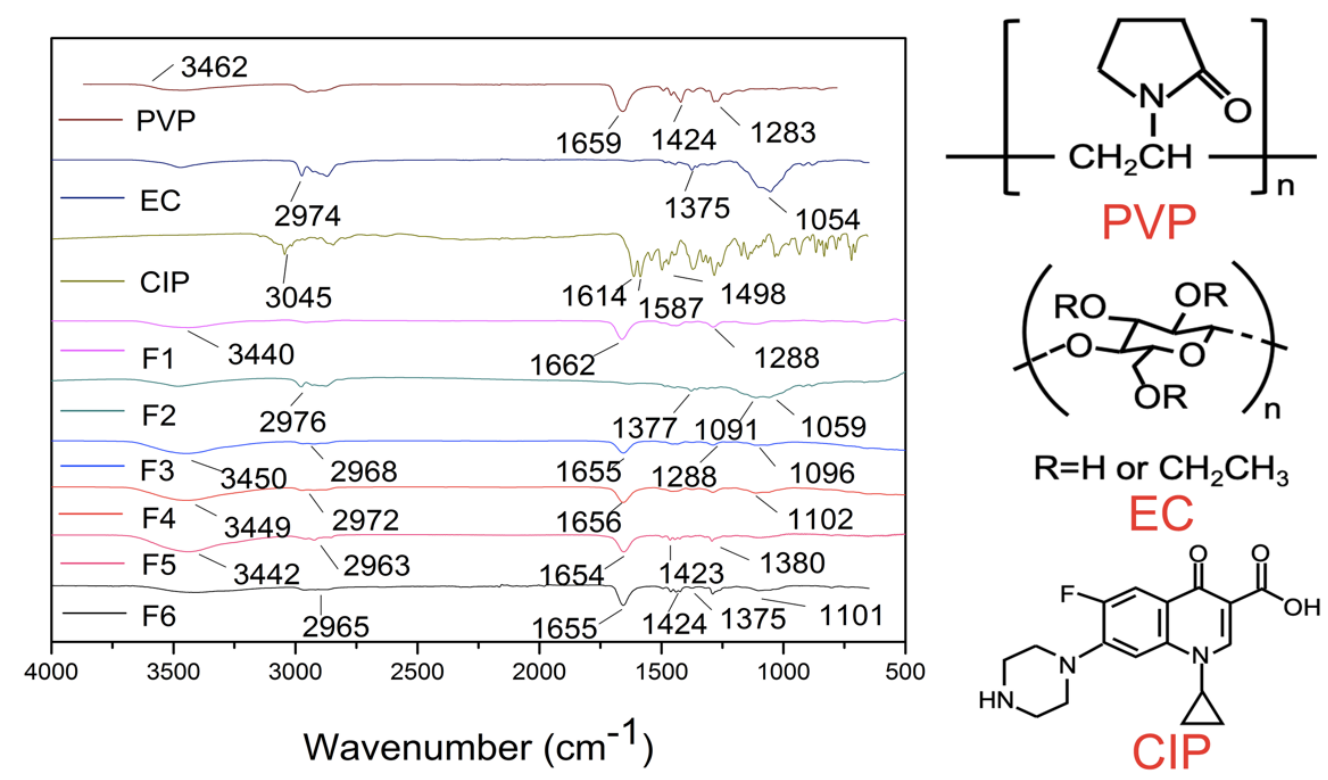

Fig. 7. FTIR spectra of the raw materials and their nanofibers and the chemical structures of the components.

\subsection{In vitro drug release profiles}

The absorbance maximum of CIP is at $276 \mathrm{~nm}$. With the predetermined calibration curve $C=0.1517 A-0.1034 \quad\left(R^{2}=0.9997\right)$, where $C$ is the concentration of CIP $\left(\mu \mathrm{g} \mathrm{m} L^{-1}\right)$, and $\mathrm{A}$ is the solution absorbance at $276 \mathrm{~nm}$, the amount of CIP released from the nanofibers can be analyzed by UV spectroscopy. 
The in vitro CIP release profiles of the monolithic fibers F1, Janus fibers F4 and F6 and raw CIP powder are depicted in Fig. 8. During the drug release tests, nanofibers F1 disappeared immediately after being placed in PBS. The in vitro tests showed that CIP released more than $80 \%$ in the first 30 s. The release behaviors of F4 and F6 are similar. Although CIP release from these Janus fibers was slower than $\mathrm{Fl}$, it was still more than $90 \%$ in the initial $30 \mathrm{~min}$. The reason for this can be attributed to the hydrophobicity of EC in other side of the Janus nanostructure. In contrast, in the first $30 \mathrm{~min}$, less than $20 \%$ of the drug was freed from the raw CIP powders.

It is widely acknowledged that a continuous antibacterial effect is beneficial in the wound healing process, which requests that the drug is release in a controlled linear manner after an initial treatment. However, almost all the monolithic medicated nanofibers release the loaded drug in a gradually decrease way. Here, the designed combination of CIP and AgNPs and the initial release can well meet the therapeutic requirements of a wound. Although a poorly water-soluble active ingredient of CIP, its fast release can provide the antibacterial effect for the treated wound. This property can be attributed to the following three aspects: 1) the hydrophilic of PVP; 2) the unique properties of electrospun nanofibers such as small diameter, huge porosity and 3D web structures; and 3) the amorphous state of CIP within the PVP matrix, as demonstrated by the above-mentioned XRD patterns. Thus, no strange that the Janus fibers can provide an immediate release of CIP for 
ensuring the great antibacterial effect at the critical treatment time of the wound healing.

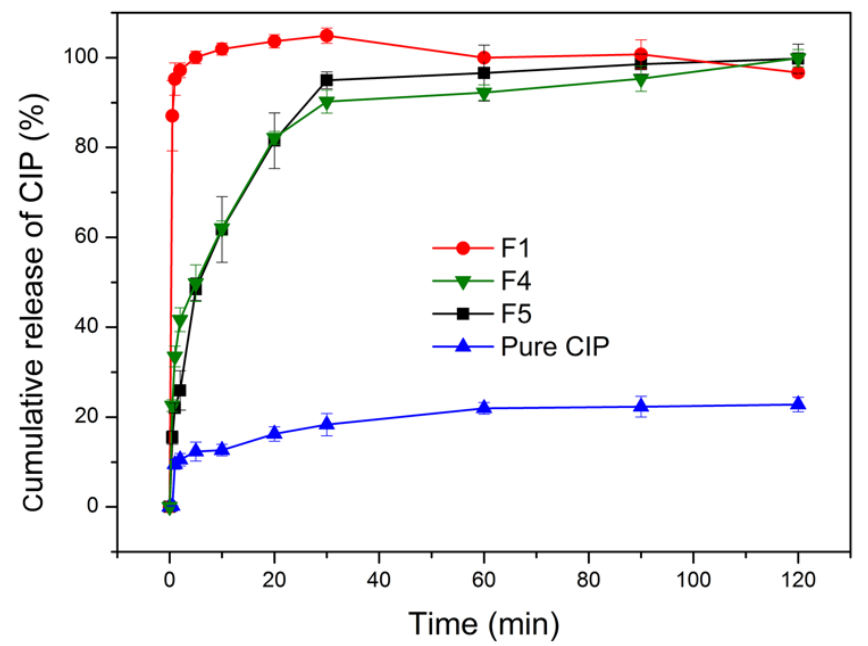

Fig. 8. The in vitro release profiles of CIP from the drug-loaded fibers and pure drug particles.

\subsection{Antibacterial activity}

The wounds are always infected by bacterial species, especially in the first 24h. Antibacterial test of the wound dressing is necessary. As a fluoroquinolone, CIP is one of the most extensively used antibiotics due to its minimal inhibitory concentration for the bacteria. And CIP also has the low frequency of spontaneous resistance [57]. The bactericidal effects of AgNPs have been observed since ancient times and it has been confirmed that AgNPs have wide-ranging effects on a broad spectrum of Gram-positive, Gramnegative and even antibiotic-resistant bacteria [58]. Fig. 9 shows the results of antibacterial tests for the PVP-CIP//EC-AgNPs wound dressings. The differences of the inhibition zones among the nanofibers can be seen clearly. 
After the agar plates incubation at $37^{\circ} \mathrm{C}$, for both $\mathrm{S}$. aureus and E. coli, no inhibition zone can be seen around the CIP/AgNPs-free PVP//EC fibers F3, indicating that polymer matrices themselves had no antibacterial activity against the bacteria. The observation of an obvious inhibition zone around the CIP-loaded F4, AgNPs-loaded F5 and CIP-PVP//EC-AgNPs F6 fibers showed that they had good antibacterial activity. The sizes of the inhibition zones are summarized in Table 2.

Comparisons of the experimental results between $24 \mathrm{~h}$ and $72 \mathrm{~h}$ for F4, F5 and F6 were undertaken. For F4, there are no significant differences in the diameters of the inhibition zones $(p>0.05)$, suggesting that the antibacterial effect of nanofibers remained stable level owing to their being no sustained release of CIP. The initially potent antibacterial effect of F4 could however be very promising for wound healing applications. In contrast, the initial antibacterial effect of $F 5$ is smaller than F4, but the F5 fibers provide a sustained antibacterial effect owing to the AgNPs on the EC side. This can be seen from the significant difference of the inhibition zone diameters $(p<0.05)$. For F6, the inhibition zone diameters between 24 and $72 \mathrm{~h}$ showed a significant difference $(p<0.05)$. These Janus products had both strong initial and an extended antibacterial effect, suggesting a synergistic action of both the CIP and AgNPs in the two sides. It can be concluded that the Janus F6 fibers show clear advantages over other products, in that they were able to provide both potent initial action and then a long-time sustained anti-bacterial effect. 
This superior performance is a direct result of the synergistic effect of CIP and AgNPs in the two sides of the Janus nanostructures and their controlled release profiles.

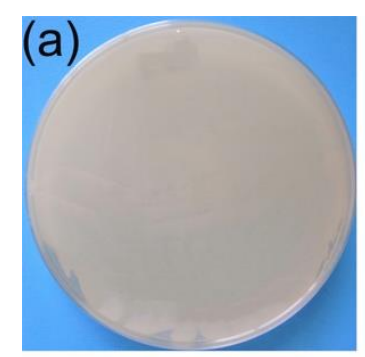

(b)

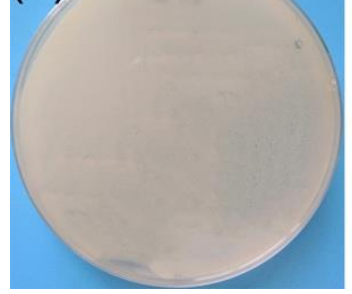

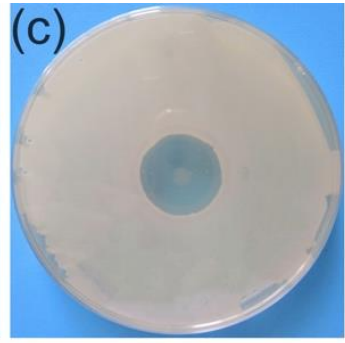

(d)

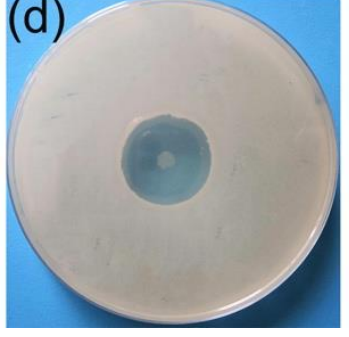

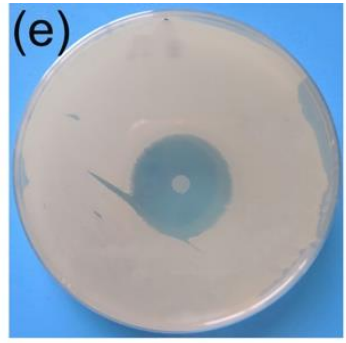

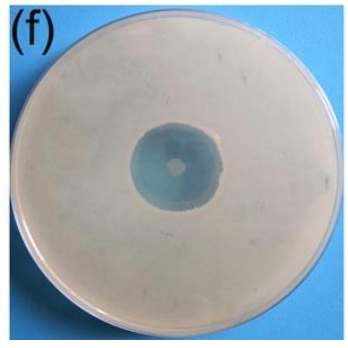

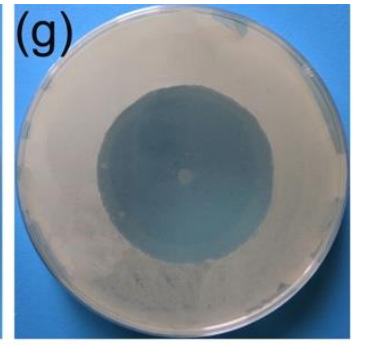

(h)

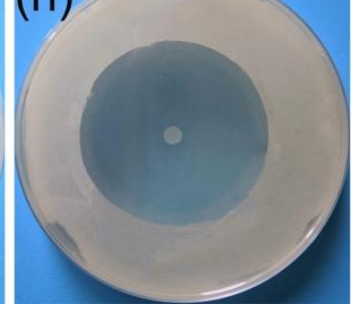

Fig. 9. Bacterial tests of selected fiber samples. (a) F3 against S. aureus; (b) F3 against E. coli; (c) F4 against S. aureus; (d) F4 against E. coli; (e) F5 against S. aureus; (f) F5 against E. coli; (g) F6 against S. aureus; (h) F6 against E. coli.

Table 2. Diameters of the fibers inhibition zone. Data are reported as mean \pm S.D. from 3 independent experiments.

Sampl Inhibition zones against S. aureus Inhibition zones against E. coli (mm) $\mathrm{e}$ $(\mathrm{mm})$ 


\begin{tabular}{|c|c|c|c|c|c|c|}
\hline F3 & --- & --- & --- & --- & --- & --- \\
\hline \multirow[t]{2}{*}{ F4 } & $11.42 \pm 0.8$ & $11.69 \pm 0$. & $12.09 \pm 0.33$ & $12.43 \pm 1.0$ & $12.96 \pm 0.3$ & $13.64 \pm 0.6$ \\
\hline & 9 & 66 & & 4 & 3 & 8 \\
\hline \multirow[t]{2}{*}{ F5 } & $9.70 \pm 0.92$ & $11.35 \pm 1$. & $14.65 \pm 0.90$ & $10.35 \pm 0.6$ & $12.05 \pm 0.9$ & $14.08 \pm 0.3$ \\
\hline & & 25 & & 4 & 5 & 3 \\
\hline \multirow[t]{2}{*}{ F6 } & $17.84 \pm 0.6$ & $18.03 \pm 0$ & $19.40 \pm 0.33$ & $21.86 \pm 0.6$ & $22.46 \pm 0.9$ & $24.04 \pm 0.3$ \\
\hline & 1 & 64 & & 2 & 8 & 3 \\
\hline
\end{tabular}

\subsection{Application mechanism}

The interests in research of wound dressing have been increased largely in the past several decades. The first electrospun wound dressing came in 2002, using Bombyx mori silk with poly (ethylene oxide), which showed an anticipated functional performance [59]. But it is until 2010, Sun et al. first reported drug-loaded wound dressing that was produced using a coaxial electrospinning process [60]. Compared with monolithic nanofiber-based wound dressings, the therapeutic effect of core-shell nanofiber-based wound dressing was significantly improved for wound healing. Here, the wound dressings comprising Janus structure via side-by-side electrospinning should perform even better. A suggested mechanism for the action of Janus fibers F6 is shown in Fig. 10. Wound is the area of skin where has a break or injury on the surface because of mechanical, thermal, physical or chemical damage. 
When the injured part returns to its original state, including appearance, structure and function, the wound is considered to be healed completely [61]. When a wound occurs, the tissues around are necrotic. And the fluids from the injured site outflow and accumulate. At that time, absorbing the exudates should be done in 15 min to avoid further bleeding, and the most urgent measure is to prevent bacterial invasion and infection [62]. The liquid absorption performance of PVP is great, so the PVP-CIP//EC-AgNPs wound dressing can quickly absorb the exudates as soon as it contacts the wound. It has been broadly reported that CIP is a broad-spectrum agent, which is able to inhibit both E. coli and S. sureus growth in the wound places $[63,64]$. The immediate release of CIP from the PVP side to the wound places ensures an initial better antibacterial activity almost at once.

Open wounds are favorable sites for the colonization of microbial. When a wound occurs, it is a polymicrobial place. Often at first, gram-positive bacteria, especially S. aureus, are predominant. Later, gram-negative bacteria E. coli is responsible for the deeper layer damage [65]. Thus, the long-time period antibacterial property is critical for a wound dressing. The antibacterial activity of AgNPs against human pathogenic bacteria has been deeply examined [66]. Around 650 species of microbes, even to the bacteria with antibiotic resistance, their powerful antimicrobial properties are widely recognized [67]. Furthermore, they can reduce the side effects of drugs which are undesirable and do not cause microbial resistance [62]. After the PVP sides 
dissolved, the EC sides are still there due to the insolubility of EC and AgNPs. The AgNPs contact directly with the bacterial cell wall. They can perform the great bacterial effect in terms of disruption of cell wall and plasma membrane components, protein deactivation, blockage of respiratory enzymatic pathway and inhibition of replication and transcription processes [68]. Thus, the ECAgNPs sides can take their roles for anti-bacterial right at the contact of Janus fibers with the wound exudates, but what important is that they can ensure a long-time period antibacterial effect until the wound place is completely healing. The synergistic action of CIP and AgNPs in the Janus nanostructures makes them candidates as potential wound dressings with excellent antibacterial functional performances.

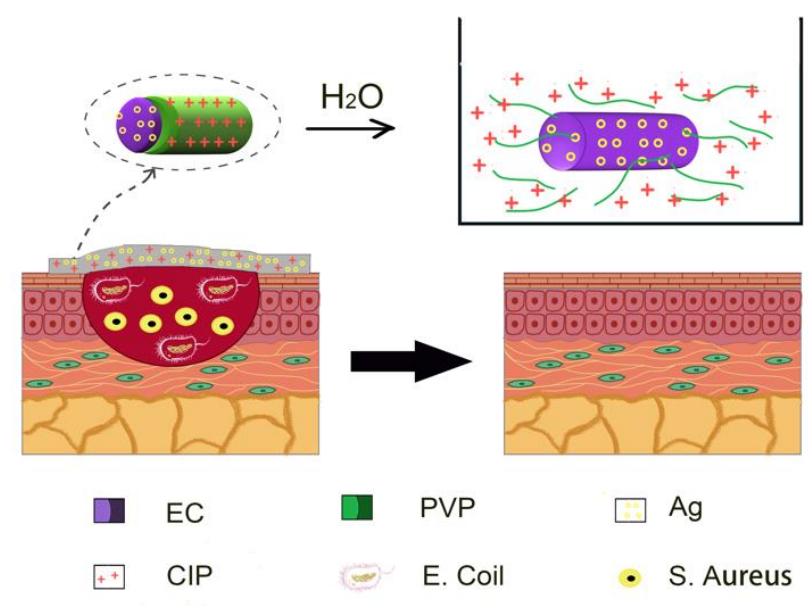

Fig. 10. Schematic diagram of skin and the application mechanism of the nanofibers. 


\section{Conclusions}

A novel wound dressing composed of PVP-CIP//EC-AgNPs hybrid Janus nanofibers was successfully prepared using a side-by-side electrospinning method employing an acentric spinneret. Images from SEM and TEM showed the smooth microscopies and uniform structures of the resultant Janus nanoproducts. XRD patterns verified that CIP and AgNPs were loaded in the Janus structures and CIP was distributed in an amorphous manner in the fibebrs owing to good chemical compatibility between CIP and PVP. In vitro dissolution tests demonstrated that the PVP-CIP sides of the Janus fibers could release over $90 \%$ of the loaded CIP within $30 \mathrm{~min}$. Antibacterial assays showed that the PVP-CIP/EC-AgNPs hybrid Janus nanofibers were able to provide more potent performance in inhibiting the growth of $\mathrm{S}$. aureus and $\mathrm{E}$. coli than other fiber formulations. The mechanism underpinning the synergistic effects from the Janus nanofibers is proposed. The PVP-CIP sides of the Janus fibers could provide a strong initial antibacterial effect due to the immediate release of CIP, and the EC-AgNPs side ensures a long-time sustained period of antibacterial action. The work reported in this study paves a new way for creating Janus nanostructure-based advanced functional materials.

\section{Acknowledgement}


This work was financially supported by the National Natural Science Foundation of China (No. 51373101 and 51803121 ).

\section{References}

[1] R. Shi, H. Geng, M. Gong, J. Ye, C. Wu, X. Hu, L. Zhang, Long-acting and broad-spectrum antimicrobial electrospun poly ( $\epsilon$-caprolactone)/gelatin micro/nanofibers for wound dressing, J. Colloid. Interface 509 (2018) 275-284. https://doi.org/10.1016/j.jcis.2017.08.092

[2] K. Yu, F. Lu, Q. Li, H. Chen, B. Lu, J. Liu, G. Lan, In situ assembly of Ag nanoparticles (AgNPs) on porous silkworm cocoon-based wound film: enhanced antimicrobial and wound healing activity, Sci. Rep. 7(1) (2017) 2107. https://doi.org/10.1038/s41598-017-02270-6

[3] Y. Loo, Y. C. Wong, E. Z. Cai, C. H. Ang, A. Raju, A. Lakshmanan, C. A. Hauser, Ultrashort peptide nanofibrous hydrogels for the acceleration of healing of burn wounds, Biomaterials 35(17) (2014) 4805-4814. https://doi.org/10.1016/j.biomaterials.2014.02.047

[4] K. Ito, A. Saito, T. Fujie, K. Nishiwaki, H. Miyazaki, M. Kinoshita, S. Takeoka, Sustainable antimicrobial effect of silver sulfadiazine-loaded nanosheets on infection in a mouse model of partial-thickness burn injury, Acta Biomater. $24 \quad$ (2015) 87-95. https://doi.org/10.1016/j.actbio.2015.05.035 
[5] D. Ye, Z. Zhong, H. Xu, C. Chang, Z. Yang, Y. Wang, L. Zhang, Construction of cellulose/nanosilver sponge materials and their antibacterial activities for infected wounds healing, Cellulose 23(1) (2016) 749-763. https://doi.org/10.1007/s10570-015-0851-4

[6] C. Gong, Q. Wu, Y. Wang, D. Zhang, F. Luo, X. Zhao, Z. Qian, A biodegradable hydrogel system containing curcumin encapsulated in micelles for cutaneous wound healing, Biomaterials 34(27) (2013) 63776387. https://doi.org/10.1016/j.biomaterials.2013.05.005

[7] H. J. Yoo, H. D. Kim, Characteristics of waterborne polyurethane/poly (Nvinylpyrrolidone) composite films for wound-healing dressings, J. Appl. Polym. 107(1) (2008) 331-338. https://doi.org/10.1002/app.26970

[8] T. Hakkarainen, R. Koivuniemi, M. Kosonen, C. Escobedo-Lucea, A. SanzGarcia, J. Vuola, M. Yliperttula, Nanofibrillar cellulose wound dressing in skin graft donor site treatment, J. Control. Release 244 (2016) 292-301. https://doi.org/10.1016/j.jconrel.2016.07.053

[9] K. A. Rieger, N. P. Birch, J. D. Schiffman, Designing electrospun nanofiber mats to promote wound healing-A review, J. Mater. Chem. B 1(36) (2013) 4531-4541. https://doi.org/10.1039/C3TB20795A

[10] R. Singla, S. Soni, V. Patial, P. M. Kulurkar, S. K. Yadav, Cytocompatible anti-microbial dressings of syzygium cumini cellulose nanocrystals decorated with silver nanoparticles accelerate acute and diabetic wound 
healing, Sci. Rep. 7(1) (2017) 10457. https://doi.org/10.1038/s41598017-08897-9

[11] J. Boateng, O. Catanzano, Advanced therapeutic dressings for effective wound healing-a review, J. Pharm. Sci. 104(11) (2015) 36533680.https://doi.org/10.1002/jps.24610

[12] K. Wang, H. F. Wen, D. G. Yu, Y. Yang, D. F. Zhang, Electrosprayed hydrophilic nanocomposites coated with shellac for colon-specific delayed drug delivery, Mater. Design 143 (2018) 248-255. https://doi.org/10.1016/j.matdes.2018.02.016

[13] Z. Liu, L. L. Zhang, Y. Y. Yang, D. Wu, G. Jiang, D. G. Yu, Preparing composite nanoparticles for immediate drug release by modifying electrohydrodynamic interfaces during electrospraying, Powder Technol. 327 (2018) 179-187. https://doi.org/10.1016/j.powtec.2017.12.066

[14] K. Zhao, W. Wang, Y. Yang, K. Wang, D. G. Yu, From Taylor cone to solid nanofiber in tri-axial electrospinning: Size relationships, Results Phys. 102270 (2019). https://doi.org/10.1016/j.rinp.2019.102770

[15] Y. Yang, T. Zhu, Z. P. Liu, M. Luo, D. G. Yu, S.W. Annie Bligh, The key role of straight fluid jet in predicting the drug dissolution from electrospun nanofibers, Int. J. Pharm. $569 \quad$ (2019) 118634. https://doi.org/10.1016/j.ijpharm.2019.118634

[16] M. Liu, X. P. Duan, Y. M. Li, D. P. Yang, Y. Z. Long, Electrospun nanofibers 
for wound healing, Mater. Sci. Eng. C-Mater. Boil. Appl. 76 (2017) 14131423. https://doi.org/10.1016/j.msec.2017.03.034

[17] Q. Niu, B. Chen, J. Guo, J. Nie, X. Guo, G. Ma, Flexible, porous, and metalheteroatom-doped carbon nanofibers as efficient ORR electrocatalysts for Zn-Air battery, Nano-Micro Lett. 11(1) (2019) 8. https://xs.scihub.Itd/https://doi.org/10.1007/s40820-019-0238-4

[18] S. P. Miguel, R. S. Sequeira, A. F. Moreira, C. C. Cabral, A. G. Mendonça, P. Ferreira, I. J. Correia, An overview of electrospun membranes loaded with bioactive molecules for improving the wound healing process, Eur. J. $\begin{array}{llll}\text { Pharm. } & \text { Biopharm. } & 139 & \text { (2019) }\end{array}$ https://doi.org/10.1016/j.ejpb.2019.03.010

[19] M. Wang, K. Wang, Y. Yang, Y. Liu, D. G. Yu, Electrospun environment remediation nanofibers using unspinnable liquids as the sheath fluids: A $\begin{array}{lllll}\text { review, } & \text { Polymers } & 12 & \text { (2020) }\end{array}$ https://doi.org/10.3390/polym12010103

[20] H. Qu, S. Wei, Z. Guo, Coaxial electrospun nanostructures and their applications, J. Mater. Chem. A 1(38) (2013) 11513-11528. https://doi.org/10.1039/c3ta12390a

[21] H. Zhou, Z. Shi, X. Wan, H. Fang, D. G. Yu, X. Chen, P. Liu, The relationships between the process parameters and the polymeric nanofibers fabricated using a modified coaxial electrospinning, Nanomaterials 9 (2019) 843. 
https://doi.org/10.3390/nano9060843

[22] C. Yang, D. G. Yu, D. Pan, X. K. Liu, X. Wang, S. W. A. Bligh, G. R. Williams, Electrospun $\mathrm{pH}$-sensitive core-shell polymer nanocomposites fabricated using a tri-axial processes, Acta Biomater. 35 (2016) 77-86. https://doi.org/10.1016/j.actbio.2016.02.029

[23] D. G. Yu, M. Wang, X. Li, X. Liu, L.-M. Zhu, S.W. Annie Bligh, Multifluid electrospinning for the generation of complex nanostructures, Wiley Interdiscip. Rev.-Nanomed. Nanobiotechnol. $\quad$ (2019) https://doi.org/10.1002/wnan.1601

[24] K. Wang, X. K. Liu, X. H. Chen, D. G. Yu, Y. Y. Yang, P. Liu, Electrospun hydrophilic Janus nanocomposites for the rapid onset of therapeutic action of helicid, ACS Appl. Mater. Interfaces 10(3) (2018) 2859-2867. https: / / doi.org/10.1021/acsami.7b1 7663

[25] C. H. Yao, C. Y. Lee, C. H. Huang, Y. S. Chen, K. Y. Chen, Novel bilayer wound dressing based on electrospun gelatin/keratin nanofibrous mats for skin wound repair, Mater. Sci. Eng. C-Mater. Boil. Appl. 79 (2017) 533-540 https://doi.org/10.1016/j.msec.2017.05.076

[26] X. Yang, L. Fan, L. Ma, Y. Wang, S. Lin, F. Yu, H. Wang, Green electrospun Manuka honey/silk fibroin fibrous matrices as potential wound dressing, Mater. Des. $119 \quad$ (2017) 76-84. https://doi.org/10.1016/j.matdes.2017.01.023 
[27] M. Wang, T. Hai, Z. Feng, D. G. Yu, Y. Yang, S. W. A. Bligh, The relationships between the working fluids, process characteristics and products from the modified coaxial electrospining of zein, Polymers 11(8) (2019) 1287. https://doi.org/10.3390/polym 11081287

[28] W. Huang, Y. Hou, X. Lu, Z. Gong, Y. Yang, X. J. Lu, X. L. Liu, D. G. Yu. The process-property-performance relationship of medicated nanoparticles prepared by modified coaxial electrospraying, Pharmaceutics 11(5) (2019) 226. https://doi.org/10.3390/pharmaceutics 11050226

[29] P. B. Tsekova, M. G. Spasova, N. E. Manolova, N. D. Markova, I. B. Rashkov, Electrospun curcumin-loaded cellulose acetate/polyvinylpyrrolidone fibrous materials with complex architecture and antibacterial activity, Mater. Sci. Eng. C-Mater. Boil. Appl. 73 (2017) 206-214. https://doi.org/10.1016/j.msec.2016.12.086

[30] N. Aghamohamadi, N. S. Sanjani, R. F. Majidi, S. A. Nasrollahi, Preparation and characterization of Aloe vera acetate and electrospinning fibers as promising antibacterial properties materials, Mater. Sci. Eng. C-Mater. Boil. Appl. 94 (2019) $445-452$. https://doi.org/10.1016/j.msec.2018.09.058

[31] M. Shahhosseininia, S. Bazgir, M. D. Joupari, Fabrication and investigation of silica nanofibers via electrospinning, Mater. Sci. Eng. C-Mater. Boil. Appl. 91 (2018) 502-511. https://doi.org/10.1016/j.msec.2018.05.068 
[32] P. He, Q. Zhong, Y. Ge, Z. Guo, J. Tian, Y. Zhou, C. Zhou, Dual drug loaded coaxial electrospun PLGA/PVP fiber for guided tissue regeneration under control of infection, Mater. Sci. Eng. C-Mater. Boil. Appl. 90 (2018) 549556. https://doi.org/10.1016/j.msec.2018.04.014

[33] B. Lu, T. Li, H. Zhao, X. Li, C. Gao, S. Zhang, E. Xie, Graphene-based composite materials beneficial to wound healing, Nanoscale 4(9) (2012) 2978-2982. https://doi.org/10.1039/C2NR1 1958G

[34] A. C. Alavarse, F. W. de Oliveira Silva, J. T. Colque, V. M. da Silva, T. Prieto, E. C. Venancio, J. J. Bonvent, Tetracycline hydrochloride-loaded electrospun nanofibers mats based on PVA and chitosan for wound dressing, Mater. Sci. Eng. C-Mater. Boil. Appl. 77 (2017) 271-281. https:// doi.org/10.1016/j.msec.2017.03.199

[35] G. Perumal, S. Pappuru, D. Chakraborty, A. M. Nandkumar, D. K. Chand, M. Doble, Synthesis and characterization of curcumin loaded PLAHyperbranched polyglycerol electrospun blend for wound dressing applications, Mater. Sci. Eng. C-Mater. Boil. Appl. 76 (2017) 1196-1204. https://doi.org/10.1016/j.msec.2017.03.200

[36] S. Kobsa, N. J. Kristofik, A. J. Sawyer, A. L. Bothwell, T. R. Kyriakides, W. M. Saltzman, An electrospun scaffold integrating nucleic acid delivery for treatment of full-thickness wounds, Biomaterials 34(15) (2013) 38913901. https://doi.org/10.1016/j.biomaterials.2013.02.016 
[37] S. Houshyar, G. S. Kumar, A. Rifai, N. Tran, R. Nayak, R. A. Shanks, A. Bhattacharyya, Nanodiamond/poly- $\epsilon$-caprolactone nanofibrous scaffold for wound management, Mater. Sci. Eng. C-Mater. Boil. Appl. 100 (2019) 378-387. https://doi.org/10.1016/j.msec.2019.02.110

[38] R. Singh, F. Ahmed, P. Polley, J. Giri, Fabrication and characterization of core-shell nanofibers using a next-generation airbrush for biomedical applications, ACS Appl. Mater. Interfaces 10(49) (2018) 41924-41934. https: / / doi.org/ 10.1021 /acsami.8b13809

[39] Q. Zhang, J. H. Oh, C. H. Park, J. H. Baek, H. M. Ryoo, K. M. Woo, Effects of dimethyloxalylglycine-embedded poly ( $\epsilon$-caprolactone) fiber meshes on wound healing in diabetic rats, ACS Appl. Mater. Interfaces 9(9) (2017) 7950-7963. https://doi.org/10.1021/acsami.6b15815

[40] J. I. Kim, H. R. Pant, H. J. Sim, K. M. Lee, C. S. Kim, Electrospun propolis/polyurethane composite nanofibers for biomedical applications, Sci. Eng. C-Mater. Boil. Appl. 44 (2014) 52-57. https: / / doi.org/10.1080/00914037.2017.1376200

[41] H. S. Jung, M. H. Kim, J. Y. Shin, S. R. Park, J. Y. Jung, W. H. Park, Electrospinning and wound healing activity of $\beta$-chitin extracted from cuttlefish bone, Carbohydr. Polym. $193 \quad$ (2018) 205-211. https://doi.org/10.1016/j.carbpol.2018.03.100

[42] J. H. Guo, G. W. Skinner, W. W. Harcum, P.E. Barnum, Pharmaceutical 
applications of naturally occurring water-soluble polymers, Drug Discov. Today 1(6) (1998) 254-261. https://doi.org/10.1016/S1461$5347(98) 00072-8$

[43] S. Prasertmanakit, N. Praphairaksit, W. Chiangthong, N. Muangsin, Ethyl cellulose microcapsules for protecting and controlled release of folic acid, AAPS PharmSciTech 10(4) $\quad 1104$. https: / / doi.org/10.1208/s12249-009-9305-3

[44] X. Ma, Y. Xiao, H. Xu, K. Lei, M. Lang, Preparation, degradation and in vitro release of ciprofloxacin-eluting ureteral stents for potential antibacterial application, Mater. Sci. Eng. C-Mater. Boil. Appl. 66 (2016) 92-99. https://doi.org/10.1016/j.msec.2016.04.072

[45] H. Li, G. R. Williams, J. Wu, Y. Lv, X. Sun, H. Wu, L. M. Zhu, Thermosensitive nanofibers loaded with ciprofloxacin as antibacterial wound dressing materials, Int. J. Pharm. 517(1-2) (2017) 135-147. https://doi.org/10.1016/j.ijpharm.2016.12.008

[46] F. L. He, X. Deng, Y. Q. Zhou, T. D. Zhang, Y. L. Liu, Y. J. Ye, D. C. Yin, Controlled release of antibiotics from poly- $\epsilon$-caprolactone/polyethylene glycol wound dressing fabricated by direct writing melt electrospinning, $\begin{array}{llll}\text { Polym. Adv } & \text { Technol. 30(2) 425-434. }\end{array}$ https://doi.org/10.1002/pat.4481

[47] X. Liu, L. H. Nielsen, S, N, Kłodzińska, H. M. Nielsen, H. Qu, L. P. 
Christensen, M. Yang, Ciprofloxacin-loaded sodium alginate/poly (lacticco-glycolic acid) electrospun fibrous mats for wound healing, Eur. J. $\begin{array}{llll}\text { Pharm. } & \text { Biopharm. } 123 & \text { (2018) } & \text { 42-49. }\end{array}$ https://doi.org/10.1016/j.ejpb.2017.11.004

[48] M. R. Reithofer, A. Lakshmanan, A. T. Ping, J. M. Chin, C. A. Hauser, In situ synthesis of size-controlled, stable silver nanoparticles within ultrashort peptide hydrogels and their anti-bacterial properties, Biomaterials 35(26) (2014) 7535-7542. https://doi.org/10.1016/j.biomaterials.2014.04.102

[49] H. Qin, H. Cao, Y. Zhao, C. Zhu, T. Cheng, Q. Wang, Y. Jiang, In vitro and in vivo anti-biofilm effects of silver nanoparticles immobilized on titanium, Biomaterials $\quad 35(33) \quad$ (2014) 9114-9125. https://doi.org/10.1016/j.biomaterials.2014.07.040

[50] N. Durán, M. Durán, M. B. de Jesus, A. B. Seabra, W. J. Fávaro, G. Nakazato, Silver nanoparticles: A new view on mechanistic aspects on antimicrobial activity, Nanomed. Nanotechnol. Biol. Med. 12(3) (2016) 789-799. https://doi.org/10.1016/j.nano.2015.11.016

[51] M. Liu, G. Luo, Y. Wang, R. Xu, Y. Wang, W. He, J. Wu, Nano-silverdecorated microfibrous eggshell membrane: processing, cytotoxicity assessment and optimization, antibacterial activity and wound healing, Sci. Rep. 7(1) (2017) 436. https://doi.org/10.1038/s41598$017-00594-x$ 
[52] R. Singla, S. Soni, V. Patial, P. M. Kulurkar, A. Kumari, S. Mahesh, S. K. Yadav, Cytocompatible anti-microbial dressings of Syzygium cumini cellulose nanocrystals decorated with silver nanoparticles accelerate acute and diabetic wound healing, Sci. Rep.7(1) (2017) 10457. https://doi.org/10.1038/s41 598-017-08897-9

[53] C. You, Q. Li, X. Wang, P. Wu, J. K. Ho, R. Jin, C. Han, Silver nanoparticle loaded collagen/chitosan scaffolds promote wound healing via regulating fibroblast migration and macrophage activation, Sci. Rep. 7(1) (2017) 10489. https://doi.org/10.1038/s41598-017-10481-0

[54] D. G. Yu, M. Zhang, Z. B. Zheng, Y. Y. Zhang, L. L. Zhang, A side-by-side spinneret, spinning device and spinning method with a round outer shape and an inner bisection capillary. Chinese Patent No: CN106381531B, 2018-08-28. (In Chinese)

[55] D. G. Yu, X. Wang, X. Y. Li, Y. Xu, C. Yan,. An Acentric Side-by-Side Spinning Head and Its Application. Chinese Patent No: CN104611773A, 2017-01-04. (In Chinese )

[56] D. G. Yu, J. J. Li, M. Zhang, G. R. Williams, High-quality Janus nanofibers prepared using three-fluid electrospinning, Chem. Commun. 53(33) (2017) 4542-4545. https://doi.org/10.1039/C7CC01661 A

[57] D. J. Mason, E. G. Power, H. Talsania, I. Phillips, V. A. Gant, Antibacterial action of ciprofloxacin, Antimicrob. Agents CH. 39(2) (1995) 2752-2758. 
https://doi.org/10.1 128/aac.39.12.2752

[58] V. Bui, D. Park, Y. C. Lee, Chitosan combined with $\mathrm{ZnO}, \mathrm{TiO}_{2}$ and $\mathrm{Ag}$ nanoparticles for antimicrobial wound healing applications: a mini review of the research trends, Polymers 9(1) (2017) 21. https://doi.org/10.3390/polym9010021

[59] H. J. Jin, S. V. Fridrikh, G. C. Rutledge, D. L. Kaplan, Electrospinning Bombyx mori silk with poly (ethylene oxide), Biomacromolecules, 3(6) (2002) 1233-1239. https://doi.org/10.1021/bm025581u

[60] B. Sun, S. Li, H. Zhang, H. Li, C. Zhao, X. Yuan, Y. Cui, Controlled release of Berberine Chloride by electrospun core/shell PVP/PLCL fibrous membranes, Int. J. Mat Prod. Tech. 37(3-4) (2010) 338-349. https://doi.org/10.1504/ijmpt.2010.031433

[61] A. Chaudhari, K. Vig, D. Baganizi, R. Sahu, S. Dixit, V. Dennis, S. Pillai, Future prospects for scaffolding methods and biomaterials in skin tissue engineering: a review, Int. J. Mol. Sci. 17(12) (2016) 1974. https://doi.org/10.3390/ijms 17121974

[62] C. L. Baum, C. J. Arpey, Normal cutaneous wound healing: clinical correlation with cellular and molecular events, Dermatol. Surg. 31(6) (2005) 674-686. https://doi.org/10.1111/j.1524-4725.2005.31612

[63] X. L. Cao, Q. H. Zhang, X. H. Pan, Mechanochemical synthesis of nano- 
ciprofloxacin with enhanced antibacterial activity, Inorg. Chem. Commun. 102 (2019) 66-69. https://doi.org/10.1016/j.inoche.2019.02.015

[64] A. Kamble, V. Shetty, S. M. Shendokar, Synthesis, Characterization and Antibacterial activity of ciprofloxacin loaded electrospun gelatin nanofibers, J. Bionanoscience, 12(5) (2018) 715-720. https://doi.org/10.1166/jbns.2018.1574

[65] A. F. Cardona, S. E. Wilson, Skin and soft-tissue infections: A critical review and the role of telavancin in their treatment, Clin. Infect. Dis. 61 (2015) S69-S78. https://doi.org/10.1093/cid/civ528

[66] M, Saravanan, S. Arokiyaraj, T. Lakshmi, Synthesis of silver nanoparticles from Phenerochaete chrysosporium (MTCC-787) and their antibacterial activity against human pathogenic bacteria, Microb. Pathog. 117 (2018) 68-72. https://doi.org/10.1016/j.micpath.2018.02.008

[67] S. Tang, J. Zheng, Antibacterial activity of silver nanoparticles: structural effects, Adv. Healthc. Mater. 7(13) (2018) 1701503. https://doi.org/10.1002/adhm.201701503

[68] C. L. Santos, A. J. R. Albuquerque, F. C. Sampaio, D. Keyson, Nanomaterials with anti- microbial properties: Applications in health sciences, Microbial Pathogens and Strategies for Combating them: Science, Technology and Education 4 (2013) 2. 
